\title{
Valorizing fish canning industry by-products to produce $\omega-3$ compounds and biodiesel
}

\author{
T. Lopes da Silva ${ }^{\text {a,* }}$, A.R. Santos ${ }^{a}$, R. Gomes ${ }^{\text {b }}$, A. Reis ${ }^{\text {a }}$ \\ a Unidade de Bioenergia, Laboratório Nacional de Energia e Geologia, Lisbon, Portugal \\ b AllMicroalgae-Secil, Lisbon, Portugal
}

\section{H I G H L I G H T S}

- An inexpensive strategy was used to valorize fish canning industry by products.

- Urea complexation was more efficient than winterization.

- The solid fraction contained $79.94 \%$ $(w / w)$ of saturated and monounsaturated FAME.

- The liquid fraction was further analysed by preparative HPLC analysis.

- After HPLC analysis, an oil fraction containing $89.25 \%$ EPA $(w / w)$ was obtained.

\section{GRAPHICAL ABSTRACT}

FISH WASTES OIL VALORISATION FOR $\omega-3$ COMPOUNDS AND BIODIESEL PRODUCTION

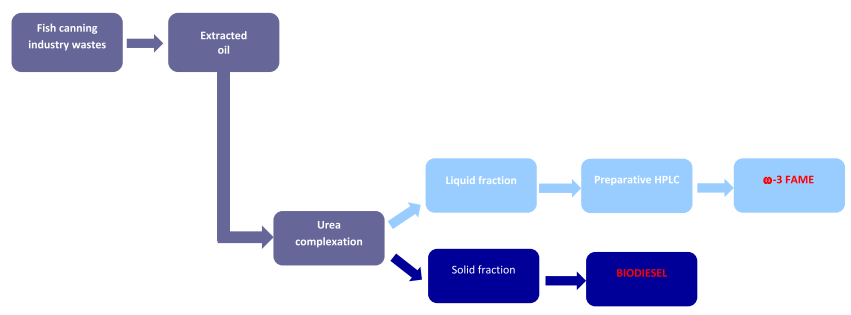

\section{A R T I C L E I N F O}

\section{Article history:}

Received 21 April 2017

Received in revised form 9 October 2017

Accepted 7 November 2017

Available online 15 November 2017

\section{Keywords:}

Fish canning wastes

Urea complexation

$\omega-3$ compounds

Biodiesel

\begin{abstract}
A B S T R A C T
A novel, inexpensive strategy was used to valorize fish canning industry by-products, aiming at $\omega-3$ compounds and biodiesel production. Winterization and urea complexation methods were used to fraction the oil extracted from fish canning industry wastes. Urea complexation was the most efficient method, producing a liquid fraction with $99.99 \%$ $(w / w)$ of polyunsaturated fatty acid methyl esters (FAME) when methanol was used as solvent, and a solid fraction with $79.94 \%(\mathrm{w} / \mathrm{w}$ ) of saturated and monosaturated FAME when ethanol was used as solvent, which was considered a potential feedstock for biodiesel production. The liquid fraction was further analysed by preparative HPLC analysis, producing an oil fraction with $99.44 \%(w / w) \omega-3$ compounds, containing $89.25 \%$ eicosapentaenoic acid (EPA) $(w / w)$. The method here described has an enormous potential for fish canning industry waste valorization as it is easily scale-up, foreseeing an environmentally sustainable society.
\end{abstract}

(c) 2017 Elsevier B.V. All rights reserved.

\footnotetext{
* Corresponding author.

E-mail address: teresa.lopessilva@lneg.pt (T. Lopes da Silva).
} 